\title{
Effect of Side Group on Mean-Square Radius of Gyration of Atactic Poly(methyl methacrylate) Chain
}

\author{
Haizhu $\mathrm{MA}^{\dagger}$ and Linxi ZHANG* \\ Department of Mathematics-Physics, Zhejiang Institute of Education, Hangzhou \\ 310012, People's Republic of China \\ * Departmant of Physics, Hangzhou University, Hangzhou \\ 310028, People's Republic of China \\ (Received May 16, 1997)
}

\begin{abstract}
Taking account of the effect of the large side group, the expression of the unperturbed mean-square radius of gyration, $\left\langle S^{2}\right\rangle$, is improved from the Lagrange relation. Using the derived relation, the ratio curve, $\left\langle S^{2}\right\rangle / x v s$. the logarithm of degree of polymerization $x$ for the atactic poly(methyl methacrylate) (A-PMMA) chain, gives a small bump, which agrees better with experiment data. The characteristic ratio $\left\langle S^{2}\right\rangle / 2 x l^{2}$ and the temperature coefficient $\mathrm{d} \ln \left\langle S^{2}\right\rangle / \mathrm{d} T$ dependence on the stereoirregularity are also investigated by the derived formula.

KEY WORDS Rotational Isomeric State / Mean-Square Radius of Gyration / Atactic Poly(methyl metacrylate) Chain /
\end{abstract}

Configuration-dependent properties for many substituted polymer chains are treated widely by the rotational isomeric state (RIS) ${ }^{1}$ theory. Among such properties, the unperturbed mean-square radius of gyration $\left\langle S^{2}\right\rangle$ is the important one, through which we can know the relation between the experimental results and the chain structure. But, the Lagrange relation of $\left\langle S^{2}\right\rangle^{2}$ is obtained only under neglecting the mass and dimension of side group. If the side group is samll, the effect of side group on $\left\langle S^{2}\right\rangle$ is insignificant. However, if the side group includes a large number of atoms, the effect is rather important. Recently, on account of the effect of side group, we have improved the calculation of $\left\langle S^{2}\right\rangle$ for the monosubstituted polymer and the symmetrically di-substituted polymer chains, ${ }^{3-5}$ which is necessary particularly to treat $\left\langle S^{2}\right\rangle$ of the short chain with large side group. Following our previous approach, we will investigate $\left\langle S^{2}\right\rangle$ of the asymmetrically di-substituted polymer chain.

The characteristic ratios for the atactic poly(methyl methacrylate) (A-PMMA) chain are widely investigated by experiment ${ }^{6.7}$ and theoretical method, including the 2-state, 3-state, and 6-state RIS models. ${ }^{8-10}$ In the experiment data of A-PMMA chain, the ratio of the mean-square radius of gyration, $\left\langle S^{2}\right\rangle / x$, passes through a maximum at a less degree of polymerization $x$ before reaching its asymptotic value at larger $x .^{6}$ However, none of the theoretical values in three RIS models has been found the small bump. ${ }^{6}$ In the treatment of ref 6 , taking account of the effect of the chemical nature of terminal groups on $\left\langle S^{2}\right\rangle / x$, the terminal statistical weight matrices are revised, nevertheless, the chain end correction improved the values of $\left\langle S^{2}\right\rangle \mid x$ only for $x \leqslant 6$. In fact, as there are many terminal groups for a substituted polymer chain, the terminal groups must be treated as the same as the chain end. We think that the behavior of short A-PMMA chain may be related to either the rotational isomeric states and the energy parameters in the statistical weight matrices or the large side group

\footnotetext{
† To whom all correspondence should be addressed.
}

atoms. In treatment of the Lagrange relation, the steric interaction of side groups is considered in the statistical weight matrices, however, the mass and size of large substituents are neglected. Therefore, we will improve the formula of $\left\langle S^{2}\right\rangle$ for the di-substituted polymer chains in this paper, and our treatment will consider the effect of side group including the steric interaction, dimension and mass. In this approach, the evaluated characteristic ratio and temperature coefficient of $\left\langle S^{2}\right\rangle$ for A-PMMA chain are in agreement with the experiment results.

\section{FORMULAE OF $\left\langle S^{2}\right\rangle$}

An A-PMMA chain, shown in Figure 1, may be assumed to be the model $[(\mathrm{b}) \mathrm{a}(\beta)-f]_{x}$ with $x$ monomeric units. Each unit is divided into F-type and A-.type atoms, including the side groups $\mathrm{b}$ and $\beta(\mathrm{b} \neq \beta)$ attached to the skeletal a-atom. Following the way of ref 1 and 3 , the unperturbed mean-square radius of gyration is calculated as

$$
\begin{aligned}
\left\langle S^{2}\right\rangle= & M^{-2}\left[\sum_{0 \leqslant i<j \leqslant 2 x} m_{i} m_{j}\left\langle r_{i j}^{2}\right\rangle\right. \\
& +\sum_{i=0}^{2 x} m_{i}\left(\sum_{j^{\prime}=1}^{x} m_{j^{\prime}}\left\langle r_{i j^{\prime}}^{2}\right\rangle+\sum_{j^{\prime \prime}=1}^{x} m_{j^{\prime \prime}}\left\langle r_{i j^{\prime \prime}}^{2}\right\rangle\right) \\
& +\sum_{1 \leqslant i^{\prime}<j^{\prime} \leqslant x} m_{i^{\prime}} m_{j^{\prime}}\left\langle r_{i^{\prime} j^{\prime}}^{2}\right\rangle \\
& +\sum_{1 \leqslant i^{\prime \prime}<j^{\prime \prime} \leqslant x} m_{i^{\prime \prime}} m_{j^{\prime \prime}}\left\langle r_{i^{\prime} j^{\prime \prime}}^{2}\right\rangle \\
& \left.+\sum_{i^{\prime}=1}^{x} \sum_{j^{\prime \prime}=1}^{x} m_{i^{\prime}} m_{j^{\prime \prime}}\left\langle r_{i^{\prime} j^{\prime \prime}}^{2}\right\rangle\right]
\end{aligned}
$$

where $M$ is the molecular weight of chain, $m_{i}, m_{i^{\prime}}$, and $m_{i^{\prime \prime}}$ are the masses of skeletal atom $i$ (a or $\mathrm{f}$ ), side groups $i^{\prime}(\mathrm{b})$ and $i^{\prime \prime}(\beta)$, respectively. $r_{i j}^{2}$ is the square of the distance from atom $i$ to $j$. According to the RIS theory. $r_{i j}^{3}$ can be calculated by

$$
r_{i j}^{2}=j_{\mathrm{P}} g_{i+1}^{(j-1)} j_{\mathrm{Q}}
$$


where

$$
\begin{aligned}
& j_{\mathrm{P}}=\left(\begin{array}{lllll}
1 & 0 & 0 & 0 & 0
\end{array}\right), \quad j_{\mathrm{Q}}=\left(\begin{array}{lllll}
0 & 0 & 0 & 0 & 1
\end{array}\right)^{T} \\
& g_{k}=\left[\begin{array}{ccc}
1 & 2 L^{T} T & l^{2} \\
0 & T & L \\
0 & 0 & 1
\end{array}\right]_{k} \quad(k=i+1, \cdots, j-i)
\end{aligned}
$$

and

$$
L_{k}=\left(\begin{array}{lll}
l_{k} & 0 & 0
\end{array}\right)^{T}
$$

Obviously, the matrix $g_{k}$ is a function of the transformation matrix $T_{k}$ and the bond length $l_{k}$. To account for the chiral order of groups attached to the skeletal aatoms in an asymmetric chain, the transformation matrix is divided into $T_{k}$ for the meso diad and $T_{k}^{*}$ for the recemic diad, then, $g_{k}$ is divided into the meso diad $g_{k}$ and the recemic diad $g_{k}^{*}$. If $r_{i j^{\prime}}^{2}, r_{i j^{\prime \prime}}^{2}, r_{i^{\prime} j^{\prime}}^{2}, r_{i^{\prime \prime} j^{\prime \prime}}^{2}$, and $r_{i^{\prime} j^{\prime \prime}}^{2}$ in eq 1 are expressed in form of the $g$ matrix, they may be unified as

$$
r_{\mathrm{L} . \mathrm{R}}^{2}=j_{\mathrm{P}} g_{\mathrm{L}} g_{i+1}^{(j-i)} g_{\mathrm{R}} j_{\mathrm{Q}}
$$

where $g_{\mathrm{L}}$ and $g_{\mathrm{R}}$ denote the left and right terminal matrices respectively in the portion of chain, and are expressed as

$$
\left.\begin{array}{lll}
g_{\mathrm{R}}=g_{\mathrm{a}} g_{\mathrm{b}}(\text { for } \mathrm{f}-\mathrm{a}-\mathrm{b}), & g_{\mathrm{R}}=g_{\alpha} g_{\beta}(\text { for } \mathrm{f}-\mathrm{a}-\beta) \\
g_{\mathrm{L}}=g_{\mathrm{e}} g_{\mathrm{f}}(\text { for } \mathrm{b}-\mathrm{a}-\mathrm{f}), & g_{\mathrm{L}}=g_{\gamma} g_{\delta}(\text { for } \beta-\mathrm{a}-\mathrm{f})
\end{array}\right\}
$$

Correspondingly, the transformation matrices $T_{k}$ and $T_{k}^{*}$ are modified by

$$
\begin{aligned}
& T_{k}=\left[\begin{array}{ccc}
\cos \theta & \sin \theta & 0 \\
\sin \theta \cos \left(\varphi+\varphi^{\prime}\right) & -\cos \theta \cos \left(\varphi+\varphi^{\prime}\right) & -\sin \left(\varphi+\varphi^{\prime}\right) \\
\sin \theta \sin \left(\varphi+\varphi^{\prime}\right) & -\cos \theta \sin \left(\varphi+\varphi^{\prime}\right) & \cos \left(\varphi+\varphi^{\prime}\right)
\end{array}\right]_{k} \\
& \left.\begin{array}{rl} 
& =T\left(\theta_{k}, \varphi_{k}+\varphi_{k}^{\prime}\right) \\
T_{k}^{*} & =T_{k} \operatorname{diag}(1,1,-1)
\end{array}\right\}
\end{aligned}
$$

where $\theta_{k}(k=\mathrm{a}, \mathrm{f}, \mathrm{c}, \mathrm{e}, \alpha, \delta, \gamma)$ is a supplement of the bond angle for the atom $k \cdot \varphi_{k}$ is the angle of internal rotation of skeletal bond $l_{k}$, and $\varphi_{k}^{\prime}$ is introduced in the terminal matrix for the reason that the transformation matrix between the skeletal bond and the side group is different from the transformation matrix between the skeletal bonds, which has been described in detail by our previous papers. $^{3,4}$ Then, the transformation matrix of F-type is divided into $T_{\mathrm{f}}, T_{\mathrm{A}}$, and $T_{\mathrm{F}}$, the A-type into $T_{\mathrm{a}}, T_{\alpha}$, and $T_{\mathrm{A}}$, they are given by

$$
\begin{array}{ll}
T_{\mathrm{A}}=T\left(\theta_{\mathrm{a}}, \varphi_{\mathrm{a}}\right), & T_{\mathrm{F}}=T\left(\theta_{\mathrm{f}}, \varphi_{\mathrm{f}}\right) \\
T_{\mathrm{f}}=T\left(\theta_{\mathrm{f}}^{\prime}, \varphi_{\mathrm{f}}+\varphi_{\mathrm{f}}^{\prime}\right), & T_{\delta}=T\left(\theta_{\delta}, \varphi_{\mathrm{f}}+\varphi_{\delta}^{\prime}\right) \\
T_{\mathrm{a}}=T\left(\theta_{\mathrm{a}}^{\prime}, \varphi_{\mathrm{a}}+\varphi_{\mathrm{a}}^{\prime}\right), & T_{\alpha}=T\left(\theta_{\alpha}, \varphi_{\mathrm{a}}+\varphi_{\alpha}^{\prime}\right) \\
T_{\mathrm{e}}=T\left(\theta_{\mathrm{e}}, \varphi_{\mathrm{e}}^{\prime}\right), & T_{\gamma}=T\left(\theta_{\gamma}, \varphi_{\gamma}^{\prime}\right)
\end{array}
$$

where $\theta_{\mathrm{a}}^{\prime}, \theta_{\alpha}, \theta_{\mathrm{e}}, \theta_{\gamma}$, and $\theta_{\mathrm{c}}$ are $\pi-\angle \mathrm{fab}, \pi-\angle \mathrm{fa} \beta$, $\pi-\angle$ baf, $\pi-\angle \beta$ af, and $\pi-\angle$ ba $\beta$, respectively. $\theta_{\mathrm{f}}^{\prime}$ and $\theta_{\delta}$ are denoted in Figure 1. $\varphi_{\mathrm{a}}^{\prime}, \varphi_{\mathrm{f}}^{\prime}, \varphi_{\mathrm{e}}^{\prime}, \varphi_{\alpha}^{\prime}, \varphi_{\delta}^{\prime}$, and $\varphi_{\gamma}^{\prime}$, may be obtained from the equations mentioned in our previous calculation $^{4}$

$$
\begin{aligned}
\cos \varphi_{\mathrm{a}}^{\prime} & =-\left(\cos \theta_{\mathrm{c}}+\cos \theta_{\mathrm{a}}^{\prime} \cos \theta_{\alpha}\right) / \sin \theta_{\mathrm{a}}^{\prime} \sin \theta_{\alpha} \\
& =\Phi\left(\theta_{\mathrm{c}}, \theta_{\mathrm{a}}^{\prime}, \theta_{\alpha}\right) \\
\cos \varphi_{\mathrm{f}}^{\prime} & =\Phi\left(\theta_{\mathrm{f}}^{\prime}, \theta_{\mathrm{e}}, \theta_{\mathrm{f}}\right) \\
\cos \varphi_{\mathrm{e}}^{\prime} & =-\Phi\left(\theta_{\gamma}, \theta_{\mathrm{c}}, \theta_{\mathrm{e}}\right)
\end{aligned}
$$

$$
\begin{aligned}
& \cos \varphi_{\alpha}^{\prime}=\Phi\left(\theta_{\mathrm{c}}, \theta_{\alpha}, \theta_{\mathrm{a}}^{\prime}\right) \\
& \cos \varphi_{\delta}^{\prime}=\Phi\left(\theta_{\delta}, \theta_{\gamma}, \theta_{\mathrm{f}}\right) \\
& \cos \varphi_{\gamma}^{\prime}=-\Phi\left(\theta_{\mathrm{e}}, \theta_{\mathrm{c}}, \theta_{\gamma}\right)
\end{aligned}
$$

where $\varphi_{\mathrm{f}}^{\prime}$ and $\varphi_{\delta}^{\prime}$ must be introduced, this approach is different from that reported in ref 11 and 12. Therefore, the terminal matrix $g_{k}(k=\mathrm{a}, \mathrm{f}, \alpha, \delta)$ are different from the internal matrices $g_{\mathrm{A}}$ and $g_{\mathrm{F}}$, nevertheless, they are all given by eq $4 . g_{\mathrm{b}} j_{\mathrm{Q}}$ and $g_{\beta} j_{\mathrm{Q}}$ are only depended on the lengthes of side groups $l_{\mathrm{b}}$ and $l_{\beta}$.

According to Frisch's approach, ${ }^{11,13}$ the microstructure of an asymmetric chain with the $x$-ads constitutions is simulated by the repeating $(p+1)$-ads, where $p$ is the number of diad in a repeating motif. Moreover, the simulated results are in fair agreement with those of the Monte Carlo model when $p \geqslant 6 .{ }^{11}$ This approach indicates that there are $2^{p}$ distinguishable configurations in the subensemble of representative chains. The occurrence probability of the $i$-th sequence of the $(p+1)$-ads, $P_{i}$, is assumed to obey the statistical law. Following Allegra's method of the pseudostereochemical equilibrium, ${ }^{14}$ the statistical models, such as Bernoullian and first-order Markovian distribution, are applied to an average on $2^{p}$ configurations of the representative chains. Following this way and the RIS theory, the unperturbed meansquare radius of gyration for a di-substituted polymer chain can be derived as

$$
\left\langle S^{2}\right\rangle=M^{-2} \sum_{i=1}^{2 p} P_{i}\left[\sum_{q=1}^{13} m(q) S_{[}(q) S_{i}^{X}(q) S_{\mathrm{]}}(q)\right] / J^{*} U_{i}^{X} J
$$

where $\sum P_{i}$ is the configurational statistics of various representative chains. The second $\sum$ refers to the factors of side groups, including their shape, position and mass. The mass values $m(q)$ of atom pairs are given by $m(1)=1.0, m(2)=m(3)=m_{\mathrm{f}} m_{\mathrm{b}}, m(4)=m(5)=m_{\mathrm{a}} m_{\mathrm{b}}, m(6)=$ $m(7)=m_{\mathrm{f}} m_{\beta}, \quad m(8)=m(9)=m_{\mathrm{a}} m_{\beta}, \quad m(10)=m_{\mathrm{b}}^{2}, \quad m(11)=m_{\beta}^{2}$, $m(12)=m(13)=m_{\mathrm{b}} m_{\beta}$. Obviously, if the mass of side group will be ignored, the summation will become insignificant, the result of eq 11 is reduced to that of Flory. ${ }^{1,2}$ According to Chapter IV of ref 1 , the divider in eq 11 is the partition function for the $i$-th representative chain, in which $U_{i}$ represents the $p$-multiplication of the meso diad statistical matrix $u_{\mathrm{f}} u_{\mathrm{am}}$ and the recemic diad matrix $u_{\mathrm{f}} u_{\mathrm{ar}}$, where $u_{\mathrm{f}}$ is the interdiad statistical matrice, $u_{\mathrm{am}}$ and $u_{\mathrm{ar}}$ are the intradiad matrices. $X$ is the number of repeating $(p+1)$-ads. Similarly, the $S_{i}^{X}(q)$ is the $X$-multiplication of $S_{i}(q)$, which represents an average of product of the transformation matrices depended on the rotational states. The meso diad matrix $S_{\mathrm{m}}(q)$ in $S_{i}(q)$ of Table I is formulated by

$$
S_{\mathrm{m}}(q)=\left[\left(u_{\mathrm{f}} \otimes E_{7}\|F(q)\|\right]\left[\left(u_{\mathrm{am}} \otimes E_{7}\right)\|A(q)\|\right]\right.
$$

and on the chain ends

$$
S_{\mathrm{I}}(q)=J^{*} \otimes J_{\mathrm{P}}\left\|A_{1}(q)\right\|, \quad S_{\mathrm{]}}=\left\|F_{x}(q)\right\| J \otimes J_{\mathrm{Q}}
$$

where

$$
\begin{gathered}
J^{*}=\left(\begin{array}{llll}
1 & 0 & \cdots
\end{array}\right), \quad J=\left(\begin{array}{lllll}
1 & \cdots
\end{array}\right)^{T} \\
J_{\mathrm{P}}=\left(\begin{array}{lllllll}
1 & 0 & 0 & 0 & 0 & 0 & 0
\end{array}\right), \quad J_{\mathrm{Q}}=\left(\begin{array}{lllllll}
0 & 0 & 0 & 0 & 0 & 0 & 1
\end{array}\right)^{T}
\end{gathered}
$$

where $J^{*}$ and $J$ are the matrices of $1 \times v$ and $v \times 1$ orders for the $v$ states scheme. The $E_{7}$ denotes the unit matrix 
of $7 \times 7$ orders. The pseudodiagonal matrix $\|F(q)\|$ or $\|A(q)\|$ is obtained by placing $F(q)$ or $A(q)$ matrix for the various rotational states of the skeletal bond in diagonal array. The matrices $F(q)$ and $A(q)$ for the F-type and A-type are expressed respectively as

$$
\begin{aligned}
& F(1)=\left[\begin{array}{ccc}
1 & m_{\mathrm{a}} j_{\mathrm{P}} g_{\mathrm{F}} & m_{\mathrm{a}} m_{\mathrm{f}} l_{\mathrm{f}}^{2} \\
0 & g_{\mathrm{F}} & m_{\mathrm{f}} g_{\mathrm{F}} j_{\mathrm{Q}} \\
0 & 0 & 1
\end{array}\right] \\
& =G\left(m_{\mathrm{a}} j_{\mathrm{P}} g_{\mathrm{F}}, m_{\mathrm{a}} m_{\mathrm{f}} l_{\mathrm{f}}^{2}, g_{\mathrm{F}}, m_{\mathrm{f}} g_{\mathrm{F}} j_{\mathrm{Q}}, \cdots\right) \\
& F(2)=G\left(0,0, g_{\mathrm{F}}, 0, \cdots\right)=F(4)=F(6)=F(8) \\
& F(3)=G\left(j_{\mathrm{p}} g_{\mathrm{e}} g_{\mathrm{f}}, r_{\mathrm{bf}}^{2}, g_{\mathrm{F}}, g_{\mathrm{F}} j_{\mathrm{Q}}, \cdots\right) \text { for } \mathrm{b}-\mathrm{a}-\mathrm{f} \cdots \mathrm{f} \\
& F(5)=G\left(j_{\mathrm{P}} g_{\mathrm{e}} g_{\mathrm{f}}, 0, g_{\mathrm{F}}, j_{\mathrm{Q}}, \cdots\right) \quad \text { for } \mathrm{b}-\mathrm{a}-\mathrm{f} \cdots \mathrm{a} \\
& F(7)=G\left(j_{\mathrm{P}} g_{i} g_{\delta}, r_{\beta \mathrm{f}}^{2}, g_{\mathrm{F}}, g_{\mathrm{F}} j_{\mathrm{Q}}, \cdots\right) \quad \text { for } \beta-\mathrm{a}-\mathrm{f} \cdots \mathrm{f} \\
& F(9)=G\left(j_{\mathrm{P}} g_{\gamma} g_{\delta}, 0, g_{\mathrm{F}}, \mathrm{j}_{\mathrm{Q}}, \cdots\right) \quad \text { for } \beta-\mathrm{a}-\mathrm{f} \cdots \mathrm{a} \\
& F(10)=G\left(j_{\mathrm{P}} g_{\mathrm{e}} g_{\mathrm{f}}, 0, g_{\mathrm{F}}, 0, \cdots\right)=F(12) \quad \text { for } \mathrm{b}-\mathrm{a}-\mathrm{f} \cdots \\
& F(11)=G\left(j_{\mathrm{P}} g_{\gamma} g_{\delta}, 0, g_{\mathrm{F}}, 0, \cdots\right)=F(13) \text { for } \beta-\mathrm{a}-\mathrm{f} \cdots
\end{aligned}
$$

$$
\begin{array}{ll}
A(1)=G\left(m_{\mathrm{f}} j_{\mathrm{P}} g_{\mathrm{A}}, m_{\mathrm{a}} m_{\mathrm{f}} l_{\mathrm{a}}^{2}, g_{\mathrm{A}}, m_{\mathrm{a}} g_{\mathrm{A}} j_{\mathrm{Q}}, \cdots\right) \\
A(2)=G\left(j_{\mathrm{P}} g_{\mathrm{A}}, r_{\mathrm{fb}}^{2}, g_{\mathrm{A}}, g_{\mathrm{a}} g_{\mathrm{b}} j_{\mathrm{Q}}, \cdots\right) & \text { for } \mathrm{a} \cdots \mathrm{f}-\mathrm{a}-\mathrm{b} \\
A(3)=G\left(0,0, g_{\mathrm{A}}, 0, \cdots\right)=A(5)=A(7)=A(9) & \text { for } \mathrm{f} \cdots \mathrm{f}-\mathrm{a}-\mathrm{b} \\
A(4)=G\left(j_{\mathrm{P}}, l_{\mathrm{b}}^{2}, g_{\mathrm{A}}, g_{\mathrm{a}} g_{\mathrm{b}} j_{\mathrm{Q}}, \cdots\right) & \text { for } \mathrm{a} \cdots \mathrm{f}-\mathrm{a}-\beta \\
A(6)=G\left(j_{\mathrm{P}} g_{\mathrm{A}}, r_{\mathrm{f} \beta}^{2}, g_{\mathrm{A}}, g_{\alpha} g_{\beta} j_{\mathrm{Q}}, \cdots\right) & \text { for } \mathrm{f} \cdots \mathrm{f}-\mathrm{a}-\beta
\end{array}
$$

Table I. Probability function of Bernoullian for the representative chain with the repeating period of magnitude $p=4$

\begin{aligned} \multicolumn{1}{r}{$i$} & $S_{i}\left[S_{i}=U_{i}, S_{i}(q)\right] \\ 1 & S_{\mathrm{m}} S_{\mathrm{m}} S_{\mathrm{m}} S_{\mathrm{m}} \\ 2 & S_{\mathrm{m}} S_{\mathrm{m}} S_{\mathrm{m}} S_{\mathrm{r}} \\ 3 & S_{\mathrm{r}} S_{\mathrm{m}} S_{\mathrm{m}} S_{\mathrm{m}} \\ 4 & S_{\mathrm{m}} S_{\mathrm{r}} S_{\mathrm{m}} S_{\mathrm{m}} \\ 5 & S_{\mathrm{m}} S_{\mathrm{m}} S_{\mathrm{r}} S_{\mathrm{m}} \\ 6 & S_{\mathrm{m}} S_{\mathrm{m}} S_{\mathrm{r}} S_{\mathrm{r}} \\ 7 & S_{\mathrm{r}} S_{\mathrm{r}} S_{\mathrm{m}} S_{\mathrm{m}} \\ 8 & S_{\mathrm{r}} S_{\mathrm{m}} S_{\mathrm{m}} S_{\mathrm{r}} \\ 9 & S_{\mathrm{m}} S_{\mathrm{r}} S_{\mathrm{r}} S_{\mathrm{m}} \\ 10 & S_{\mathrm{m}} S_{\mathrm{r}} S_{\mathrm{m}} S_{\mathrm{r}} \\ 11 & S_{\mathrm{r}} S_{\mathrm{m}} S_{\mathrm{r}} S_{\mathrm{m}} \\ 12 & S_{\mathrm{r}} S_{\mathrm{r}} S_{\mathrm{r}} S_{\mathrm{m}} \\ 13 & S_{\mathrm{m}} S_{\mathrm{r}} S_{\mathrm{r}} S_{\mathrm{r}} \\ 14 & S_{\mathrm{r}} S_{\mathrm{m}} S_{\mathrm{r}} S_{\mathrm{r}} \\ 15 & S_{\mathrm{r}} S_{\mathrm{r}} S_{\mathrm{m}} S_{\mathrm{r}} \\ 16 & S_{\mathrm{r}} S_{\mathrm{r}} S_{\mathrm{r}} S_{\mathrm{r}}\end{aligned}$

$$
\begin{array}{ll}
A(10)=G\left(0,0, g_{\mathrm{A}}, g_{\mathrm{a}} g_{\mathrm{b}} j_{\mathrm{Q}}, \cdots\right)=A(13) & \text { for } \cdots \mathrm{f}-\mathrm{a}-\mathrm{b} \\
A(11)=G\left(0,0, g_{\mathrm{A}}, g_{\alpha} g_{\beta} j_{\mathrm{Q}}, \cdots\right)=A(12) & \text { for } \cdots \mathrm{f}-\mathrm{a}-\beta
\end{array}
$$

In all matrices above, $\cdots$ denote the elements of 0 or 1 , their position in each metrix is the same. The recemic diad matrix $S_{\mathrm{r}}(q)$ in $S_{i}(q)$ is obtained by replacing $g_{\mathrm{F}}, g_{\mathrm{f}}$, and $u_{\mathrm{am}}$ with $g_{\mathrm{F}}^{*}, g_{\mathrm{f}}^{*}$, and $u_{\mathrm{ar}}$ in $S_{\mathrm{m}}(q)$. When the lengthes $l_{\mathrm{b}}$ and $l_{\beta}$ of side groups are ignored, eq 11 is reduced to the Lagrange relation. ${ }^{1.2}$

\section{RESULTS FOR ATACTIC POLY(METHYL METHACRYLATE) CHAIN}

A-PMMA chain has the substitutents $-\mathrm{COOCH}_{3}$ and $-\mathrm{CH}_{3}$, and the mass of substitutents is $74 / 26$ of that of the skeletal group. The $b$ in Figure 1 is located at the mass centre of ester methyl $-\mathrm{COOCH}_{3}, l_{\mathrm{b}}$ is the length of virtual bond. Using the geometrical parameters in Table II of ref $8, l_{b}$ is $2.422 \AA$. Let $\theta_{a}^{\prime \prime}$ be $\pi-$ $\angle \mathrm{CC}^{\alpha} \mathrm{C}^{*}$, the supplements of virtual bond angles $\theta_{\mathrm{a}}^{\prime}$ and $\theta_{\mathrm{f}}^{\prime}$ are estimated by

$$
\begin{aligned}
& \cos \theta_{\mathrm{a}}^{\prime}=\cos (\delta+\alpha) \sin \left(\theta_{\mathrm{a}} / 2\right) \\
& \cos \delta=\cos \theta_{\mathrm{a}}^{\prime \prime} / \sin \left(\theta_{\mathrm{a}} / 2\right) \\
& \cos \theta_{\mathrm{f}}^{\prime}=\cos \theta_{\mathrm{f}} \sin \left(\theta_{\mathrm{f}}-\theta_{\mathrm{a}} / 2\right) \sin \left(\theta_{\mathrm{a}} / 2\right)
\end{aligned}
$$

where $\delta$ is the dihedral angle between the bond $\mathrm{C}^{\alpha}-\mathrm{C}^{*}$ and the plane of its adjacent skeletal bonds. $\alpha$ is that of between the bond $\mathrm{C}^{\alpha}-\mathrm{C}^{*}$ and $\mathrm{C}^{\alpha}-\mathrm{b}$. The angles $\varphi_{\mathrm{a}}^{\prime}, \varphi_{\mathrm{f}}^{\prime}$, $\varphi_{\alpha}^{\prime}$, and $\varphi_{\delta}^{\prime}$ are estimated by eq 10 . In this calculation, the torsion angle $\chi$ of bond $\mathrm{C}^{\alpha}-\mathrm{C}^{*}$ is fixed at 0 .

According to the RIS theory, ${ }^{8.9}$ each skeletal bond of the A-PMMA chain may be assigned the rotational states, the trans state $\varphi_{\mathrm{t}}$ and the gauche state $\varphi_{\mathrm{g}}$. They are adopted by 0 and $110^{\circ}$ for the 2 -state model, and 0 and $\pm 120^{\circ}$ for the 3 -state model, respectively. The corresponding interdiad statistical weight matrice $u_{\mathrm{f}}$, and the intradiad matrices $u_{\mathrm{am}}$ or $u_{\mathrm{ar}}$ are given by eq $2-4$ of ref. 8 and eq 3-8 of ref 9 , in which the statistical weights are depended on the temperature $T$ by

$$
\eta=\eta_{0} \exp \left(E_{\eta} / R T\right) \quad(\eta=\alpha, \beta, \rho)
$$

The energy parameters $E_{\eta}$ and $\eta_{0}$ are listed in Table II. In eq 11 , the $P_{i}$ adopts the Bernoullian function of $p=4$ shown in Table $\mathrm{I}^{11}$ and the number $X$ is taken as $(x-1) / p$. Following the established equations and the parameters for the A-PMMA chain above, we calculate

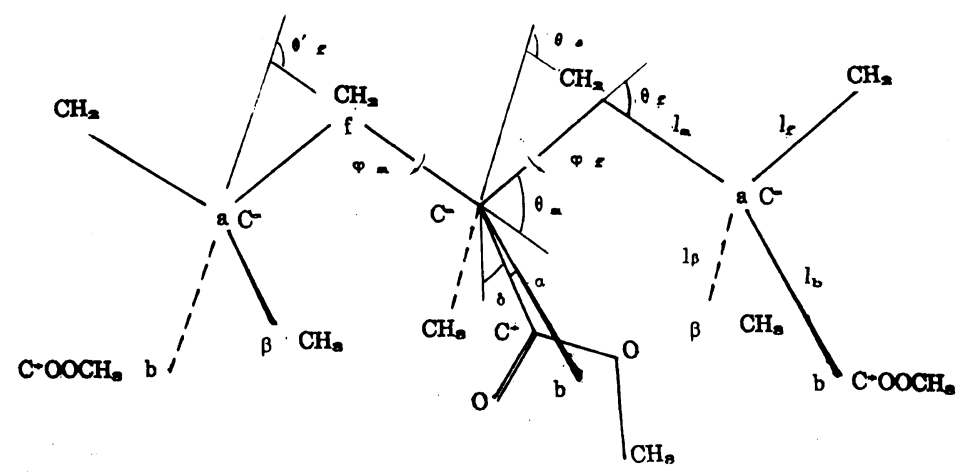

Figure 1. A segmental model of the atactic poly(methyl methacrylate) chain with a pair of side groups $\mathrm{b}$ and $\beta(\mathrm{b} \neq \beta)$. 
Table II. Parameters for the A-PMMA chain

\begin{tabular}{cccc}
\hline \multirow{2}{*}{ Scheme } & Parameter & $\eta_{0}$ & Energy $E_{\eta}$ \\
& & & $\mathrm{kcal} \mathrm{mol}^{-1}$ \\
\hline \multirow{2}{*}{ 2-State model } & $\alpha$ & 1.60 & 1.30 \\
& $\beta$ & 1.40 & -0.20 \\
3-State model & $\alpha$ & 0.85 & 1.00 \\
& $\beta$ & 0.77 & -1.00 \\
& $\rho$ & 0.87 & 1.00 \\
\hline
\end{tabular}

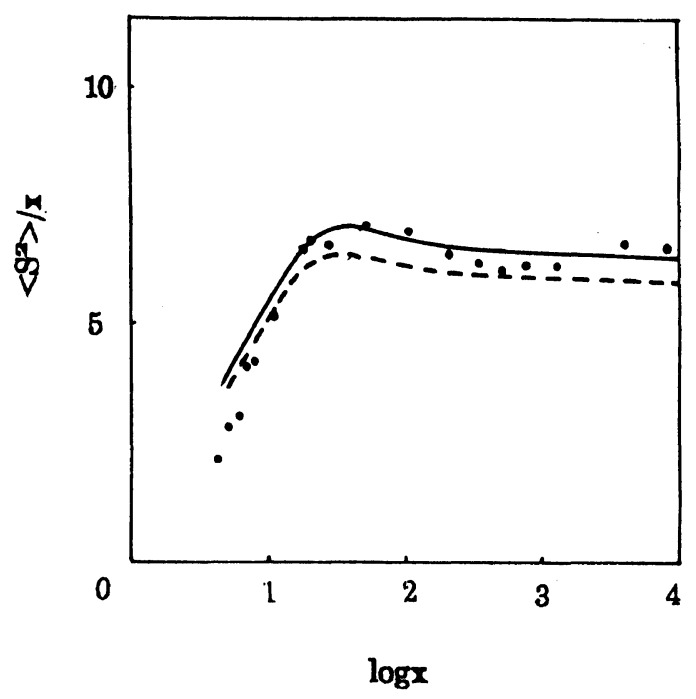

Figure 2. Dependence of ratio $\left\langle S^{2}\right\rangle / x$ on the logarithm of $x$ for the A-PMMA chain at $T=317 \mathrm{~K}$. The solid curve, the value of 2 -state model with $n_{m}=0.21$; the dashed curve, the value of 3-state model for the syndiotactic chain; the dots, the experiment data with $n_{\mathrm{m}}=0.21$ by Tamai et al.

the ratio $\left\langle S^{2}\right\rangle / x$ dependence on the logarithm of degree of polymerization $x$ at temperature $T=317 \mathrm{~K}$. In Figure 2 , the solid curve is calculated by the 2 -state model with the occurrence probability of meso diad $w_{\mathrm{m}}=0.21$, and the dashed curve is the 3 -state model for the syndiotactic chain. They are compared with the dots of the experiment data, determined by small-angle X-ray scattering and light scattering for the A-PMMA samples with $w_{\mathrm{m}}=0.21 .^{6}$ The maxima are near $x=33$, and right shift slightly as $w_{m}$ increasing. The ratio $\left\langle S^{2}\right\rangle / x$ of 2 -state model for $x>6$ are fairly close to the experiment data, which has an asymptotic value about 6.27 for larger $x$. However, there is the difference between our results and the experiment data for $x \leqslant 6$. If the chain end correction is considered following ref 6 , i.e., the 3 -state terminal statistical matrices and the large energy parameters, the increasing of the partition function is more than $\sum P_{i}$ in eq 11 , then, the $\left\langle S^{2}\right\rangle$ value will be less than the present one. Therefore, the chain end correction may be improved our results to agree with the experiment data for $x \leqslant 6$.

The characteristic ratio $\left\langle S^{2}\right\rangle / 2 x l^{2}$ as a function of $w_{m}$ is also calculated by the two states scheme in Figure 3. The curve is convex rather than the concave of the monosubstituted vinyl chains. ${ }^{5.11}$ This means that $\left\langle S^{2}\right\rangle / 2 x l^{2}$ increases with the stereoirregularity, and the enhancement of the dimensions by the stereoirregularity is com-

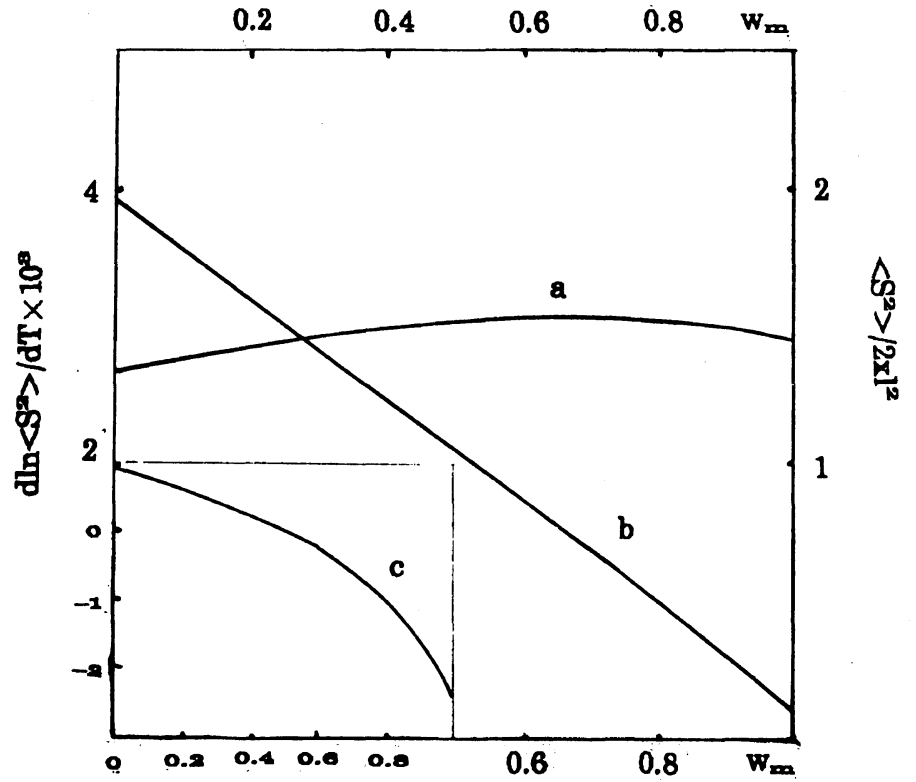

Figure 3. Curve a, the characteristic ratio $\left\langle S^{2}\right\rangle / 2 x l^{2}$ of A-PMMA chain. Curve $\mathrm{b}$ and $\mathrm{c}$, the temperature coefficient $\mathrm{d} \ln \left\langle S^{2}\right\rangle / \mathrm{d} T$ with $x=841$ and $x=37$, respectively for A-PMMA chain against the percentages of meso dyad $w_{\mathrm{m}}$ at $T=317 \mathrm{~K}$.

paratively small. The value of temperature coefficient $\mathrm{d} \ln \left\langle S^{2}\right\rangle / \mathrm{d} T$ against $w_{\mathrm{m}}$, calculated by $\sum_{i=1}^{2^{p}} P_{i} \mathrm{~d} \ln \left\langle S^{2}\right\rangle /$ $\mathrm{d} T$, is plotted in Figure 3. The curve $\mathrm{b}$ for the larger $x$ is markedly different from the curve $\mathrm{c}$ for the less $x$. The temperature coefficient of the syndiotactic chain is larger than that of the isotactic chain. This indicates that the predominantly syndiotactic poly(methyl methacrylate) chain is more extended with temperature increasing. ${ }^{8}$ The plots $\mathrm{b}$ and $\mathrm{c}$ shift down as temperature $T$ increasing in our calculation. Therefore, the extent of extension decreases as temperature increasing. Our approach is applicable to investigate the dimension of other polymer chains.

Acknowledgment. This work was supported by $\mathrm{Na}-$ tional Natural Science Foundation of China.

\section{REFERENCES}

1. P. J. Flory, "Statistical Mechanics of Chain Molecules," Interscience, New York, N.Y., 1969.

2. P. J. Flory, Macromolecules, 7, 381 (1974).

3. M. Haizhu and Z. Linxi, Pollym. J., 26, 121 (1994).

4. M. Haizhu and X. Jianmin, Polym. J., 26, 779 (1994).

5. M. Haizhu, W. Jianfan, and X. Jianmin, Eur. Polym. J., 1997. in press.

6. Y. Tamai, T. Konishi, Y. Einaga, M. Fujii, and H. Yamakawa, Macromolecules, 23, 4067 (1990).

7. R. Jenkins and R. S. Porter, Polymer, 23, 105 (1982).

8. P. R. Sundararajan and P. J. Flory, J. Am. Chem. Soc., 96, 5025 (1974).

9. P. R. Sundararaian, Macromolecules, 19, 415 (1986).

10. M. Vacatello and P. J. Flory, Macromolecules, 19, 405 (1986).

11. C. A. Givanni, Y. Deyue, and Z. Zhiping, Polymer, 34, 2830 (1993).

12. Z. Zhiping and Y. Deyue, J. Chem. Phys., 96, 4792 (1992).

13. H. L. Frisch, C. L. Mallows, F. Heatley, and F. A. Bovey, Macromolecules, 1, 533 (1968).

14. G. Allegra and S. Bruckner, Macromolecules, 10, 106 (1977). 